\title{
Pre-surgical Alveolar Molding: An Adjunct to Surgical Repair in Cleft Lip and Palate Rehabilitation
}

\author{
Rikta Pande, ${ }^{1}$ Bandana Koirala, ${ }^{2}$ Mamta Dali, ${ }^{3}$ Kabiraj Poudel ${ }^{4}$ \\ ${ }^{1}$ Junior Resident, ${ }^{2}$ Professor, ${ }^{3}$ Associate Professor, ${ }^{4}$ Consultant Pedodontist \\ ${ }^{1-3}$ Department of Pedodontics and Preventive Dentistry, College of Dental Surgery, B.P. Koirala Institute of Health Sciences, Dharan, Nepal, \\ ${ }^{4}$ Mugu District Hospital, Mugu, Nepal.
}

\begin{abstract}
Bilateral cleft lip and palate (CLP) with a severely protruded premaxillary segment can present as a challenge during surgical lip repairing. Pre-surgical alveolar molding,as described in this case report,is one such technique which aids in the approximation of the cleft segments thereby improving the esthetic result of the surgical repair. The present case highlights the importance of a simple molding appliance in re-positioning the protruded premaxillary segments of a neonate with bilateral CLP thereby serving as an adjunct to the surgical repair.
\end{abstract}

Keywords: Bilateral cleft lip and palate, lip taping, premaxillary segment, pre-surgical alveolar molding.

\section{INTRODUCTION}

Cleft lip and/or palate is the most common congenital defect of the oral cavity. ${ }^{1}$ In context of Nepal,the incidence of cleft lip and/or palate in Eastern Nepal was found to be $1.64 / 1000$ live births per year. ${ }^{2}$ Among the various types of cleft,cleft lip and palate (CLP) is the most common type. Bilateral CLP is a congenital malformation in which the premaxilla is suspended from the tip of the nasal septum along with a significantly increased alar base width. ${ }^{3}$ Bilateral CLP with a severely protruded premaxillary segment can present as a challenge during the surgical lip repairing. Pre-surgical alveolar molding, as described in this case report is one of the infant orthopedics technique which aids in the approximation of the cleft segments thereby improving the esthetic result of the surgical repair.

\section{CASE REPORT}

A 5-day-old neonate, who was born by Caesarian delivery, presented to the department of Pedodontics and Preventive

\section{Correspondence}

\section{Dr. Rikta Pande}

Junior Resident,Department of Pedodontics and Preventive Dentistry,BPKIHS,Dharan,Nepal.

E-mail: riktapande@gmail.com

\section{Citation}

Pande R, Koirala B, Dali M, K Poudel. Pre-surgical Alveolar Molding: An Adjunct to Surgical Repair in Cleft Lip and Palate Rehabilitation. J Nepal Assoc Pediatr Dent. 2021;2(1):32-5.
Dentistry,B.P. Koirala Institute of Health Sciences,with the chief complaint of inability in suckling milk and passage of fluids through the nose (Figure 1,2). There was no positive family history. Pre-natal history revealed no any illnesses, history of alcohol consumption,medication intake,trauma or hospitalization during pregnancy.

On clinical examination, bilateral complete clefts of the lip, hard and soft palate was found, with nasal deformity and a dis placed alveolar segment. Clinically, it was characterized under Veau's Class IV classification (Figure 3). ${ }^{4}$ No other associated syndromes could be appreciated.

Alveolar molding technique was planned. Both the risks and benefits were explained to the parents,but the parents seemed unwilling because of the tedious,initial follow-up schedule. However, after one month, they revisited and agreed upon undergoing the therapy. Informed parental consent was then obtained.

An impression of the cleft was taken when the infant was fully awake by using a heavy body polyvinyl siloxane impression material (Dentsply Sirona) in an acrylic custom tray (Figure 4). Impression was taken in the Pedodontics department itself with a medical officer present as a part of the impression team. While making impression, the baby was kept in his father's lap with head facing downward (inverted position) to allow the fluids to drain out of the oral cavity while the father's hands supported the baby's 


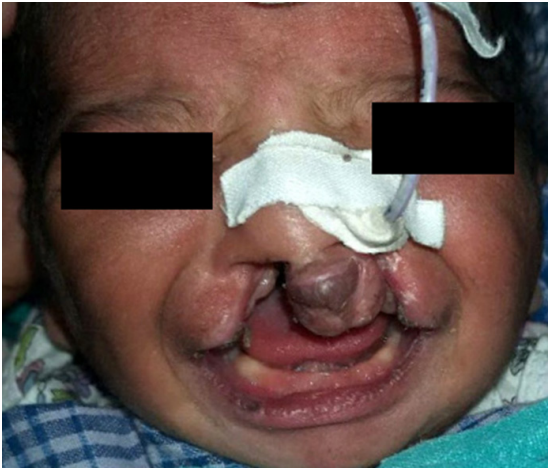

Figure 1. Extra-oral Photograph (Frontal View).

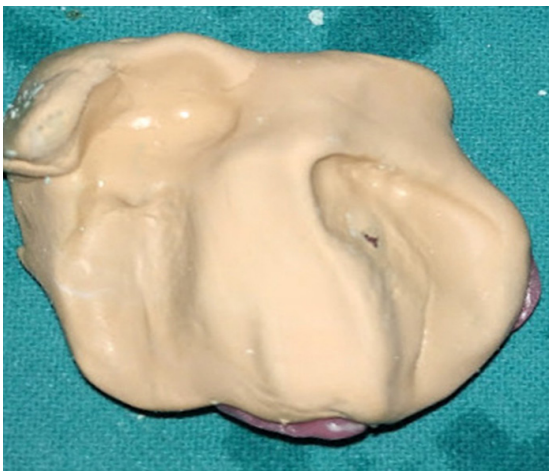

Figure 4. Primary Impression.

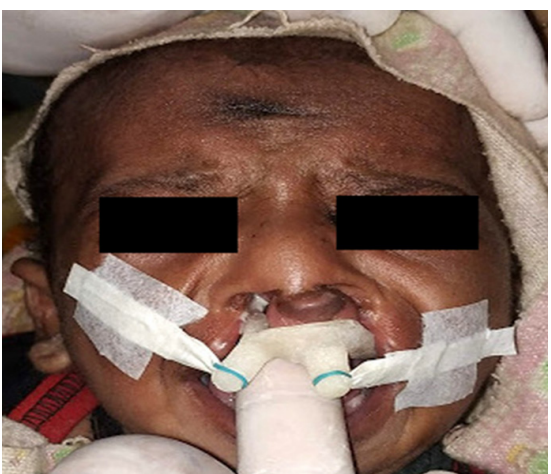

Figure 7. Appliance In Situ.

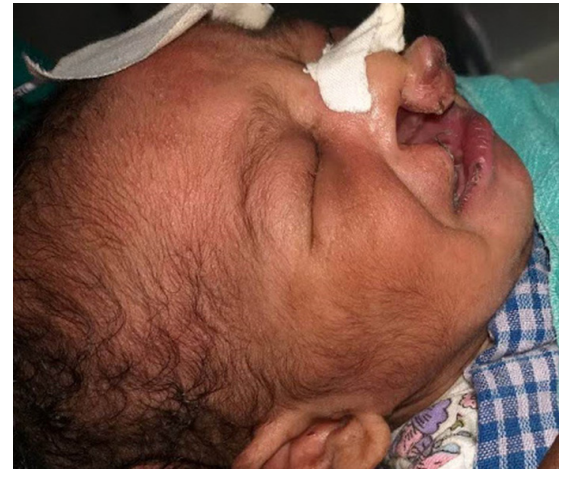

Figure 2. Extra-oral Photograph (Lateral View).

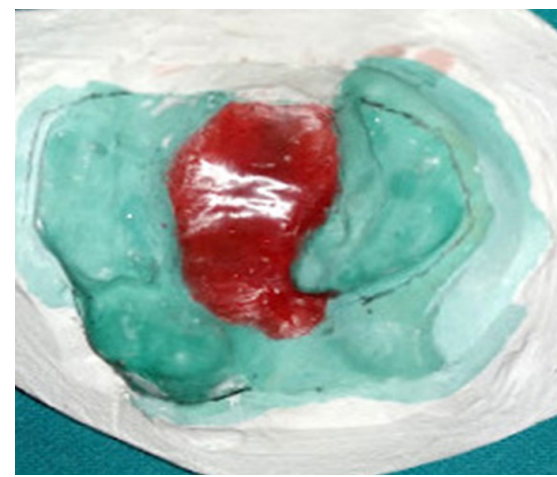

Figure 5. Preliminary Cast .

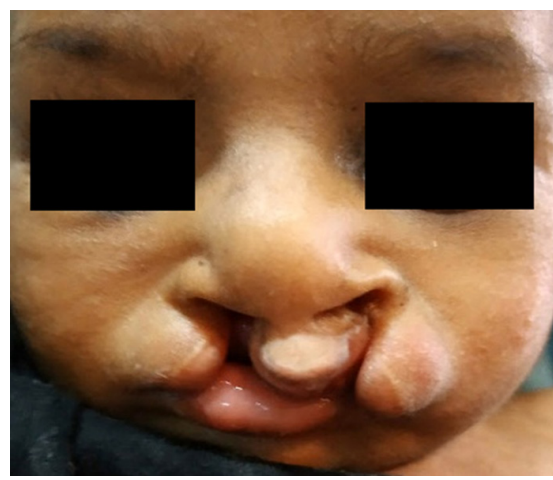

Figure 8. Post-treatment photograph taken at three months follow-up (Frontal View).

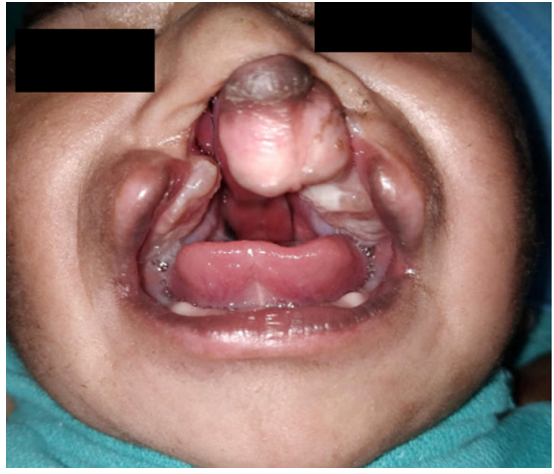

Figure 3. Veau's Class IV Cleft lip and palate.

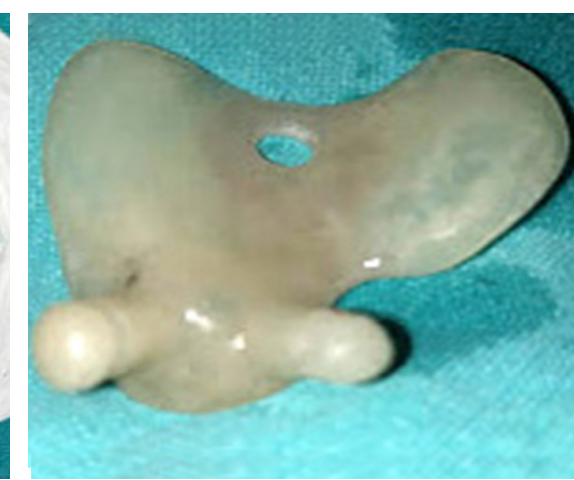

Figure 6. AlveolarMolding Plate.

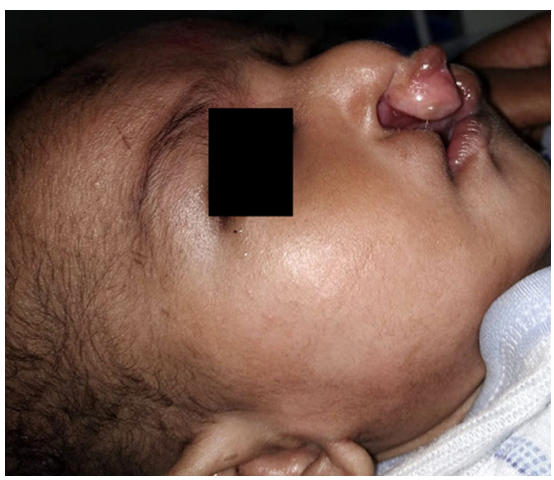

Figure 9. Post-treatment photograph taken at three months follow-up (Lateral View). chest and lap region during the entire procedure. Once the impression material was set,the tray was removed,and oral cavity was inspected for the presence of any residual impression material in the cleft region.

Cast was then poured,trimmed and an alveolar molding appliance was fabricated by using self-cure acrylic resin of 2-3 mm thickness (Figure 5,6). The appliance was finished and polished to ensure that all tissue borders were smooth and polished. A retention arm of acrylic was made at an angle of 40 degrees from the plate. A notch was created on the retention arm to grasp the elastics. The appliance was then inserted into the patient's mouth and was checked for proper fit and retention (Figure 7). The primary retention of the appliance was obtained by using extra-oral facial tapes (Micropore tape and Tegaderm) and elastics of an internal diameter 0.25 inches.

Parents were given proper instructions regarding feeding and maintenance of the appliance. They were asked and taught to feed the child with the appliance in place. The infant must be able to suckle and swallow without gagging or struggling. They were also asked to clean the appliance daily with drinking water. Parents were demonstrated 
and trained on the removal and placement of elastics and changing of tapes every day and to keep the appliance in place at all times except during cleaning.

Patient was recalled after 24 hours to evaluate and correct problems associated with the appliance (if any). Thereafter, the recall appointments were scheduled weekly for making further adjustments. During these visits, serial modification of the appliance was done by selective trimming and addition of the soft liner,depending on the direction in which the bone movement was required. The modification was done by making $0.5-1 \mathrm{~mm}$ increments during each visit.

Follow-up evaluation at three months post appliance placement showed an appreciable close approximation of cleft alveolar gap (Figure 8,9). A nasal stent was planned for the correction of nasal deformity when the cleft gap would reach approximately 5-6 $\mathrm{mm}$ but it could not be given to this patient since the infant was already four months of age. At this time,oral and maxillofacial surgeon had planned for the surgery of cleft lip thus the patient was finally sent to maxillofacial and plastic surgery team for further treatment.

\section{DISCUSSION}

Bilateral CLP is a congenital malformation where the premaxilla is often rotated to one side. The greatest challenge for reconstruction is the protruded pre-maxilla. ${ }^{3}$ If the lip segments are sutured while the pre-maxilla is still protruded,the surgical closure of the lip can be extremely difficult and due to the uncontrolled tension applied by the scarred lip, over-extrusion and bending of the premaxilla are inevitable. Here pre-surgical alveolar molding can bring about a promising result in solving many such problems of bilateral CLP to a great extent. Alveolar molding prior to primary cheiloplasty will not only provide psychological reassurance to the parents and child but also enhance the surgical outcome and reduce the need for revision surgeries in the future, thereby reducing the overall cost of the treatment. ${ }^{5}$

Pre-surgical naso-alveolar molding appliance works on the principle of 'negative sculpturing' and 'passive molding' of the alveolus and the adjacent soft tissues. ${ }^{6}$ In passive molding, a custom-made molding plate of the acrylic is used to gently direct growth of the alveolus to get the desired results later on. While in negative sculpturing, serial modifications are made in certain areas of the internal surfaces of the molding appliance, with addition or deletion of material to get the desired shape of alveolus and the nose. ${ }^{6}$

The present case reports a case of a newborn patient who had a complete bilateral cleft lip and palate and was aided by an alveolar molding plate that facilitated both the feeding as well as approximation of the cleft segments. Presurgical alveolar molding appliance provided alignment of the displaced segments, which enabled the surgeons and the patient to enjoy the benefits associated with the repair of cleft deformities with minimum number of surgeries and their complications.

The clinical procedures and fabrication of the alveolar molding plate should be started in the first week or the early second week after birth, ${ }^{1}$ as per the Matsuo and Hirose's hypothesis of Pre-surgical naso-alveolar molding which conceptualizes that the nasal cartilage is still developing and is subject to repositioning within the first 6 weeks of life. ${ }^{7}$ Molding of the tissues can be easily achieved during early life, because of raised levels of hyaluronic acid and maternal circulating estrogen levels present in the neonates. ${ }^{7}$ However, cases have been reported in which alveolar molding has been attempted even in three months old baby. ${ }^{8}$ In the present case, alveolar molding was started when the infant was 36 days old. Despite the fact that the infant reported one month late for treatment, a good treatment result could be achieved as the molding plate therapy yielded a significant reduction of cleft width in this case.

As accordance to the authors, Grayson and Shetye ${ }^{9}$ when the alveolar gap is approximated (up to five $\mathrm{mm}$ ) and the arch is aligned, a nasal molding device is added to the orthopedic appliance to increase the columella length as well as to reshape the alar dome. Following this principle, in the present case the nasal stent was not incorporated till the alveolar gap was reduced to almost five $\mathrm{mm}$. Nonetheless, there are other techniques too where alveolar and nasal molding is performed simultaneously using an acrylic plate with rigid acrylic nasal extension. ${ }^{10}$ Further studies comparing both the techniques would be helpful to better understand the results. 


\section{CONGLUSIONS}

With an aid of simple molding appliance therapy such as pre-surgical alveolar molding, a clinically visible approximation of the cleft alveolar segments was achieved along with an appreciable repositioning of the protruded pre-maxillary segment. Thus, Pre-surgical alveolar molding served as a valuable adjunct in the present case that enabled the surgeons to repair the cleft lip and palate without extensive tissue tension and the need for multiple surgeries along with great patient satisfaction.

Conflict of Interest: None

JNAPD

\section{REFERENCES}

1. Laxmikanth SM,Karagi T,Shetty A,Shetty S. Nasoalveolar molding: A review. JCRI. 2014;1(3):108-13. [Full Text | DOI]

2. Singh VP,Sagtani R,Sagtani A. Prevalence of cleft lip and cleft palate in a tertiary hospital in Eastern Nepal. Mymensingh Med J. 2012 Jan;21(1):151-4. [PubMed] Broadbent TR,Woolf RM. Cleft lip nasal deformity. Ann Plast Surg. 1984 Mar;12(3):216-34. [ [PubMed_ I DOI]

4. Allori AC,Mulliken JB,Meara JG,Shusterman S,Marcus JR. Classification of Cleft Lip/Palate: Then and Now. Cleft Palate Craniofac J. 2017 Mar;54(2):175-188. [PubMed | DOI]

5. Mathew A,Muddaiah S,Subrahmanya JB,Somaiah S, Shetty B,Reddy G. Presurgical nasoalveolar molding in a 4-day-old infant with unilateral cleft lip, alveolus, and palate deformity. APOS Trends Orthod 2018;8:225-9. [Full Text $\perp$ DOI]

6. Dubey RK,Gupta DK,Chandraker NK. Presurgical nasoalveolar molding: A technical note with case report. Indian J Dent Res Rev 2011;2:66-8. [Full Text]

7. Matsuo K,Hirose T,Tomono T,Iwasawa M,Katohda S, Takahashi N, Koh B. Nonsurgical correction of congenital auricular deformities in the early neonate: a preliminary report. Plast Reconstr Surg. 1984 Jan;73(1):38-51. [PubMed | Full Text | DOI]

8. Attiguppe PR,Karuna YM,Yavagal C,Naik SV,Deepak BM,Maganti R,Krishna CG. Presurgical nasoalveolar molding: A boon to facilitate the surgical repair in infants with cleft lip and palate. Contemp Clin Dent. 2016 Oct-Dec;7(4):569-573. [PubMed | Full Text | DOI]

9. Grayson BH,Maull D. Nasoalveolar molding for infants born with clefts of the lip,alveolus,and palate. Clin Plast Surg. 2004 Apr;31(2):149-58. [PubMed | Full $\underline{\text { Text }}$ | DOI]

10. Singh A,Thakur S, Singhal P,Diwana VK,Rani A. A Comparative Evaluation of Efficacy and Efficiency of Grayson's Presurgical Nasoalveolar Molding Technique in Patients with Complete Unilateral Cleft Lip and Palate with Those Treated with Figueroa's Modified Technique. Contemp Clin Dent. 2018 Jun;9(Suppl 1):S28-S33. [PubMed | Full Text |DOI] 